\title{
SHIELD: A Strategy-Proof and Highly Efficient Channel Auction Mechanism for Multi-radio Wireless Networks ${ }^{\star}$
}

\author{
Zuying Wei, Tianrong Zhang, Fan Wu ${ }^{\star \star}$, Guihai Chen, and Xiaofeng Gao \\ Shanghai Key Laboratory of Scalable Computing and Systems \\ Department of Computer Science and Engineering \\ Shanghai Jiao Tong University, China \\ \{zu_ying_hi, ztr1211, wu-fan,gchen,gao-xf\}@sjtu.edu.cn
}

\begin{abstract}
Due to users' fast-growing demands, wireless spectrum is becoming a more and more scarce resource. However, the state of spectrum usage shows that while large chunks of spectrum are left idle at many places, many emerging wireless applications cannot get enough spectrum to provide their services. In contrast to existing truthful mechanisms for channel redistribution, which achieve strategy-proofness at the price of lowered system performance, we propose SHIELD, which not only guarantees strategy-proofness in the process of channel redistribution, but also achieves high system performance. Our evaluation results show that SHIELD outperforms the existing mechanisms, in terms of spectrum utilization and user satisfaction ratio. Here, channel utilization represents the average number of radios allocated to channels, and buyer satisfaction ratio shows the percentage of buyers who get at least one channel in the auction.
\end{abstract}

Keywords: Wireless Network, Channel Allocation, Mechanism DesignWireless Network, Channel Allocation, Mechanism Design.

\section{Introduction}

As the fast development of the communication technologies, the spectrum is becoming a more and more scarce resource. It is expected that global mobile data traffic will increase 26-fold between 2010 and 2015 [5]. To adapt the fast growth of data traffic over wireless links, next generation wireless applications need more spectrum to carry their services. However, traditional spectrum management makes new wireless network applications face the plight of increasingly scarce spectrum resources. Currently, almost every country has a specific department for regulating spectrum usage, e.g. Federal Communications Commission (FCC) [11] in the US and Radio Administration Bureau(RAB) in China [28]. FCC and RAB divide available wireless spectrum into a number of bands, and grants the right of using each band within a specified geographical area to a particular business organization or wireless application. Traditional static spectrum allocation has been unable to meet the growing demand for wireless

\footnotetext{
* This work was supported in part by China NSF grant 61170236 and 61133006 . The opinions, findings, conclusions, and recommendations expressed in this paper are those of the authors and do not necessarily reflect the views of the funding agencies or the government.

** Corresponding author.
}

X. Wang et al. (Eds.): WASA 2012, LNCS 7405, pp. 72-87, 2012.

(C) Springer-Verlag Berlin Heidelberg 2012 
broadband services [12]. On one hand, frequency bands for wireless communications have almost been fully allocated [10]. On the other hand, already allocated spectrum is not fully utilized. For example, measurement results show that in downtown Berkeley, the utilization of spectrum up to $3 \mathrm{GHz}$ is only about $32 \%$, while for the spectrum of $3-5 \mathrm{GHz}$ the utilization is less than $1 \%$ [45]. Therefore, to improve the spectrum utilization, we need to find a more efficient mechanism to redistribute the idle spectrum to the wireless applications that need the spectrum resource.

A usual way to implement spectrum redistribution is to use auction, by which the spectrum owner (seller) gets profit through leasing idle spectrum to the wireless applications (buyers) who need the spectrum. In the literature, there are a number of auction mechanisms proposed for dynamic spectrum/channel redistribution, e.g., [39, 50,52]. These auction mechanisms target at guaranteeing strategy-proofness of the spectrum auction. Intuitively, an auction mechanism is strategy-proof, if it is the best strategy for each buyer to truthfully report her valuation of the good as the bid, no matter what the others do, and nobody's individual rationality is hurt. Two commonly used metrics for evaluating the efficiency of a spectrum auction mechanism are spectrum utilization and buyer satisfaction ratio. Here, spectrum utilization captures the average number of buyers (or radios if the buyers have multiple radios) correspond to each channel, and buyer satisfaction ratio represents the percentage of buyers who get at least one channel in the auction. Although most of existing channel auction mechanisms achieve strategyproofness, they provide low guarantee for the allocation efficiency in terms of spectrum utilization and buyer satisfaction ratio.

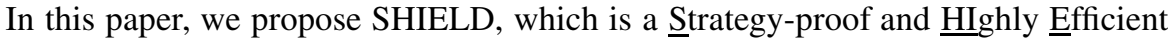
channeL auction mechanism for multi-raDio wireless networks. SHIELD not only guarantees strategy-proofness, but also achieves high performance compared with existing mechanisms. SHIELD divides the buyers into non-conflicting groups, in which every pair of buyers are well separated and can do the transmission on the same channel simultaneously, and gives larger groups higher precedence to be allocated a channel. In this paper, we make the following key contributions.

- First, we model the problem of channel redistribution as a sealed-bid auction, and propose a simple but efficient channel auction mechanism, namely SHIELD.

- Second, we prove that SHIELD is a strategy-proof channel auction mechanism.

- Third, we do extensive simulations to compare the performance of SHIELD with existing representative channel auction mechanisms, such as SMALL and VERITAS. Evaluation results verify that SHIELD guarantees strategy-proofness, and show that SHIELD outperforms existing representative channel auction mechanisms in terms of spectrum utilization and user satisfaction ratio.

We organize the rest of this paper as follows. In Section 2, we present the game model for the problem of channel redistribution and review some important solution concepts from game theory. In Section 3, we give the detailed description of SHIELD. In Section 4, we illustrate simulation results of our auction mechanism. In Section 5 , we discuss related works. In Section 6, we conclude the paper and point out potential directions for future work.

\section{Preliminaries}

In this section, we show our game-theoretic model, and review some closely related solution concepts from game theory. 


\subsection{Game-Theoretic Model}

We model the problem of channel redistribution as a sealed-bid auction, in which there are a spectrum seller and a number of buyers. The seller holds $m$ idle wireless channels, denoted by $C=\left\{c_{1}, c_{2}, \ldots, c_{m}\right\}$. The seller wants to lease her idle channels to buyers to get some profit. A channel can be leased to multiple buyers, who are not conflicting with each other according to an adequate Signal to Interference and Noise Ratio (SINR). Buyers, such as WiFi access points, desperately need the channels to serve their customers. Suppose there are $n$ buyers, denoted by $N=\{1,2, \ldots, n\}$. Each buyer has a private valuation of a channel, denoted by $v=\left\{v_{1}, v_{2}, \ldots, v_{n}\right\}$. Each buyer may equip with a single radio or multiple radios, so a buyer may bid for one or multiple channels. We assume that each channel is of the same value to each buyer. Therefore, we require that each buyer bid equally for each channel she requests. We also assume that the buyers do not cheat about the number of radios she has. Each buyer $i \in N$ has a per-channel valuation $v_{i}$. The per-channel valuation can be the revenue gained by the buyer for serving her customers. The channel valuation $v_{i}$ is private information to the buyer $i$. In the auction, each buyer $i$ submits her sealed per-channel bid $b_{i}$ together with the number of radios $r_{i}$ she has to the seller/auctioneer. The seller/auctioneer will decide auction result based on the buyers' bids. Then, we can represent the buyers' bid vector as $b=\left(b_{1}, b_{2}, \ldots, b_{n}\right)$, and demand vector as $r=\left(r_{1}, r_{2}, \ldots, r_{n}\right)$.

The seller/auctioneer use a deterministic channel allocation algorithm to determine determines the channel allocation $y=\left(y_{1}, y_{2} \ldots, y_{n}\right)$ based on the bids. Here, $y_{i}$ means that buyer $i$ gets $y_{i}$ channels in the auction. Then the $k$ th $\left(k \leq y_{i}\right)$ radio of buyer $i$ can work on the $k$ th channel allocated to $i$. Each buyer $i$ should pay for the channels she won in the auction with price $p_{i}=\sum_{k=1}^{y_{i}} p_{i}^{k}$. Here, $p_{i}^{k}$ represents the charge to buyer $i$ 's $k$ th channel. The utility $u_{i}$ of buyer $i$ is defined as the difference between her valuation of allocated channels and the charge of using the channels:

$$
u_{i}=\sum_{k=1}^{y_{i}} u_{i}^{k}=\sum_{k=1}^{y_{i}}\left(v_{i}-p_{i}^{k}\right)=v_{i} \cdot y_{i}-p_{i} .
$$

Here, $u_{i}^{k}$ represents the utility of buyer $i$ gets on the $k$ th channel. We assume that the buyers are rational, and always want to maximize their own utilities. In contrast to an individual buyer's objective, our auction mechanism aims to achieve high channel allocation efficiency, in terms of spectrum utilization and buyer satisfaction ratio.

Here, we use an example to show that traditional VCG auction model [6, 15, 33] cannot guarantee strategy-proofness for spectrum auction. VCG auction model sorts the bidders in non-increasing order, and then allocate the channels to the bidders one by one using lowest indexed channel in each bidder's available channel set. The charge to bidder $i$ is the bid of the bidder who would get the channel if bidder $i$ is absent. We model the interference among bidders using a conflict graph, which means that two bidders cannot use the same channel simultaneously if there is an edge between each other. Fig. 1 shows a simple example to illustrate that the VCG auction violates strategy-proofness. We assume that there are two channels waiting to be leased out. In Fig. 1(a), all the bidders bid truthfully and in Fig.1(b) bidder $E$ bids untruthfully. Table 1 shows the utilities of all the bidders when $E$ bids truthfully and untruthfully. We can see that when $E$ bids $b_{E}=v_{E}=4$, he loses in the auction and get utility of 0 . When he bids $b_{E}=6 \neq v_{E}$, he wins in the auction and gets the utility of 2. The bidder $E$ can increase his utility by bidding untruthfully. We can see that the traditional VCG auction model cannot guarantee strategy-proofness. 

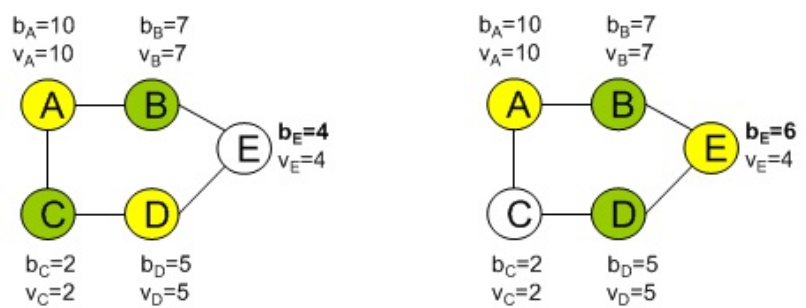

(a) User A, B, C, D get the channel. (b) User A, B, D, E get the channel.

Fig. 1. A simple example which shows that traditional VCG auction model violates strategyproofness

Table 1. Utilities of all the bidders when $E$ bids truthfully and untruthfully

\begin{tabular}{|l|l|}
\hline Fig. $[1(\mathrm{a})$ & Fig. $[1$ (b) \\
\hline$v_{A}=10, b_{A}=10, u_{A}=6$ & $v_{A}=10, b_{A}=10, u_{A}=8$ \\
\hline$v_{B}=7, b_{B}=7, u_{B}=3$ & $v_{B}=7, b_{B}=7, u_{B}=7$ \\
\hline$v_{C}=2, b_{C}=2, u_{C}=2$ & $v_{C}=2, b_{C}=2, u_{C}=0$ \\
\hline$v_{D}=5, b_{D}=5, u_{D}=1$ & $v_{D}=5, b_{D}=5, u_{D}=3$ \\
\hline$v_{E}=4, b_{E}=4, \mathbf{u}_{\mathbf{E}}=\mathbf{0}$ & $v_{E}=4, \mathbf{b}_{\mathbf{E}}=\mathbf{6}, \mathbf{u}_{\mathbf{E}}=\mathbf{2}$ \\
\hline
\end{tabular}

In Section 3.1, we will present our strategy-proof channel auction mechanismSHIELD.

\subsection{Solution Concepts}

We review two important solution concepts from game theory in this section.

Definition 1 (Dominant Strategy [14,26])

A strategy $s_{i}$ is player $i$ 's dominant strategy, if for any $s_{i}^{\prime} \neq s_{i}$ and any strategy profile of the other players $s_{-i}$, her utility satisfies:

$$
u\left(s_{i}, s_{-i}\right) \geq u\left(s_{i}^{\prime}, s_{-i}\right) .
$$

In our game model, each buyer is a player, and $s_{i}$ is a buyer $i$ 's bid.

A dominant strategy of a player is one that maximizes her utility regardless of what strategies the other players choose. Before defining strategy-proofness, we review the definition of incentive-compatibility and individual-rationality. An auction mechanism is incentive-compatible if it is one's dominant-strategy for bidding real valuation. Individual-rationality means that the buyers can always achieves at least as much utility from participating in the auction as staying outside.

\section{Definition 2 (Strategy-Proof Mechanism [24, 31])}

A mechanism is strategy-proof if it satisfies both incentive-compatibility and individualrationality. 


\section{Design and Analysis of SHIELD}

In this section, we present detailed description of our channel allocation auction mechanism, namely SHIELD. SHIELD follows the design methodology of SMALL [39]. However, with a novel winner selection method, SHIELD greatly improves spectrum utilization and buyer satisfaction ratio.

\subsection{Design of SHIELD}

We now present the design of SHIELD. SHIELD works in three steps: buyer grouping, winner selection, and charge determination.

When a buyer $i$ is equipped with $r_{i} \operatorname{radio(s),~we~use~} r_{i}$ elementary buyer(s) to represent the buyer $i$ (e.g., Fig. 2). We use $N^{\prime}$ to denote the set of elementary buyers. Therefore, each elementary buyer requests only one channel. Actually, a buyer who is equipped with one radio is an elementary buyer herself. Similar to [39, 51], SHIELD groups the buyers in a bid-independent way. SHIELD models the interference among buyers using a conflict graph. Each node in the graph is an elementary buyer. For each radio equipped by a buyer, we use an elementary buyer to represent it. Each edge in the graph represents that the two elementary buyers who interfere with each other. Since the radios belonging to the same buyer have interference between each other, we connect the nodes/elementary buyers of the same buyer with each other to indicate the confliction. There are also conflicts across nodes/elementary buyers belonging to different buyers, we connect them with each other to represent the conflicts. Then SHIELD divides all the elementary buyers into non-conflicting groups based on the conflict graph. We can use existing graph coloring algorithms (e.g., [38]) to figure out the grouping.

Fig. 2] shows a toy example. In Fig. 2] buyer $A$ is equipped with two radios. Node $A^{1}$ and $A^{2}$ represent the two elementary buyers of buyer $A$. Similarly, $B^{1}$ and $B^{2}$ represent the two elementary buyers of buyer $B, C^{1}$ and $C^{2}$ represent the two elementary buyers of buyer $C$. There are seven elementary buyers. There are many possible grouping results, for example $g_{1}=\left\{A^{1}, C^{2}\right\}, g_{2}=\left\{A^{2}, B^{1}, D\right\}$ and $g_{3}=\left\{B^{2}, C^{1}\right\}$.

Without loss of generality, we assume that the elementary buyers have been divided into $x$ non-conflicting groups by a given graph coloring algorithm:

$$
G:\left\{g_{1}, g_{2}, \ldots, g_{x}\right\} \text {. }
$$

Next, we discuss the very important step-winner selection. SHIELD sorts the buyer groups according to group size in non-increasing order as follows:

$$
G^{\prime}:\left|g_{1}^{\prime}\right| \geq\left|g_{2}^{\prime}\right| \geq \ldots \geq\left|g_{x}^{\prime}\right|
$$

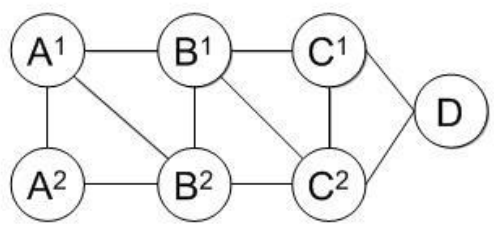

Fig. 2. A simple conflict graph 
In case of a tie, each tied group has an equal probability of being ordered prior to the others. SHIELD chooses the first $m$ (or $x$, if $x<m$ ) groups as winning groups. Furthermore, SHIELD sets the elementary buyers except the one with the smallest bid in each winning group as winners. In case of a tie, each tied elementary buyer has an equal probability of being selected as a winner. Algorithm 1 shows the pseudo code for the winner selection process.

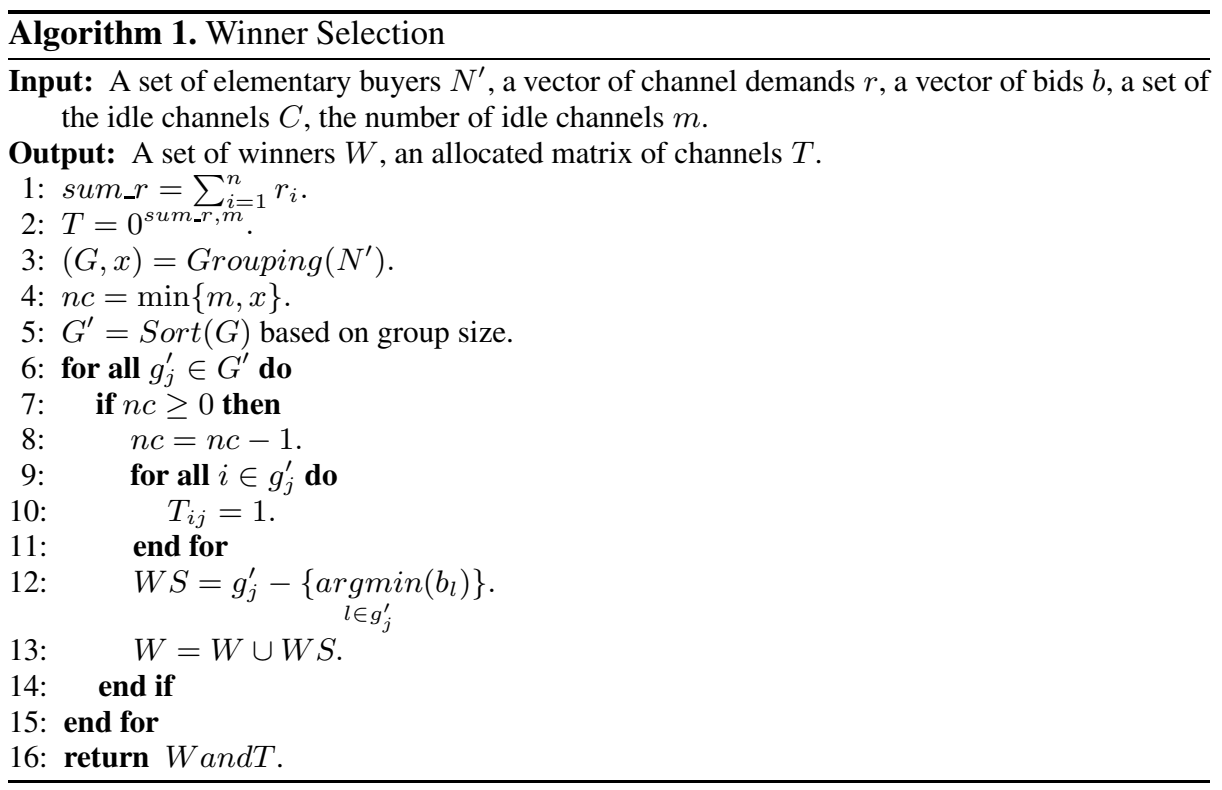

We note that to achieve strategy-proofness, SHIELD sacrifices a buyer in each winning group. No matter how large the set of buyers is, the number of sacrificed buyers is bounded by $m$, which is the number of channels for leasing.

Finally, we determine the charges to the winners. The winners in each group are charged equally and the charge is the smallest bid in that group. For each winner $l \in g_{j}^{\prime}$, the payment of her is $\min \left\{b_{q} \mid q \in g_{j}^{\prime}\right\}$. Then the charge $p_{i}$ to a buyer $i \in N$ is the sum of charges to her winning elementary buyers. We note that since each elementary buyer will not be charged more than her bid. The seller's income is the payments of all the buyers who get the channels:

$$
\text { Income }=\sum_{i=1}^{n} p_{i} .
$$

\subsection{Analysis}

In this section, we prove that SHIELD is strategy-proof, which means that reporting one's truthful per-channel valuation as a bid is the best strategy of each buyer. 
Lemma 1. When SHIELD is used, it is a dominant-strategy for each buyer to truthfully report her per-channel valuation as the bid.

Proof. SHIELD use a bid-independent grouping method to group the elementary buyers. The bid of buyer $i$ will not affect the winning group selection method. Next, we will show that no matter how a buyer bids, she can not increase her utility by bidding untruthfully. In other words, a buyer can not increase her utility by misreporting.

If buyer $i$ bids truthfully(i.e., $b_{i}=v_{i}$ ) and gets $y_{i}$ channel. Then her utility is

$$
u_{i}=\sum_{k=1}^{y_{i}} u_{i}^{k}=\sum_{k=1}^{y_{i}}\left(v_{i}-p_{i}^{k}\right) .
$$

Let $t$ be the $k$ th one of the elementary buyers generated from buyer $i$. Suppose $t \in g_{j}^{\prime}$. If $g_{j}^{\prime}$ is not a winning group, then $t$ cannot be a winner no matter how buyer $i$ bids. We then analyze the case, in which $g_{j}^{\prime}$ is a winning group. We prove that buyer $i$ can not increase her utility get from elementary buyer $t$ by bidding untruthfully. We distinguish two cases:

- If $b_{i}=v_{i}=\min \left\{b_{s} \mid s \in g_{j}\right\}$ when bidding truthfully. In this case, the elementary buyer $t$ would lose in auction or win with a charge equal to her valuation. So $u_{i}^{k}=$ 0 . Let's see the utility get from the elementary buyer $t$ if buyer $i$ bids untruthfully. We further distinguish two cases:

If the bid $b_{i}^{\prime}<b_{i}, t$ will also lose in the auction and result in the utility $u_{i}^{k}=0$.

If the bid $b_{i}^{\prime}>b_{i}$, the utility on $t$ is 0 when $t$ still loses in the auction. If $t$ wins in the auction, her utility will be $u_{i}^{\prime k}=v_{i}-p_{i}^{\prime k}$. That means the new lowest bid of the group $\min \left\{b_{s} \mid s \in g_{j} \backslash\{t\}\right\} \geq b_{i}=v_{i}$.

$$
\begin{aligned}
u_{i}^{\prime k} & =v_{i}-p_{i}^{\prime k} \\
& =v_{i}-\min \left\{b_{s} \mid s \in g_{j} \backslash\{t\}\right\} \\
& \leq v_{i}-b_{i} \\
& =0 .
\end{aligned}
$$

The utility on $t$ will be non-positive. We can get that, if $b_{i}=v_{i}=\min \left\{b_{s} \mid s \in g_{j}\right\}$, no matter how $i$ bids, she can not improve her utility got on $t$ and her utility on $t$ will be no more than 0 .

- If $b_{i}=v_{i}>\min \left\{b_{s} \mid s \in g_{j} \backslash\{t\}\right\}$, buyer $t$ would win in the auction and get the utility $u_{i}^{k}=b_{i}-p_{i}^{k}=b_{i}-\min \left\{b_{s} \mid s \in g_{j} \backslash\{t\}\right\}$ if $i$ reports her bid truthfully. If $i$ bids untruthfully, the utility of the buyer will be also $u_{i}^{k}$ or will become 0 .

Assume $i$ bids $b_{i}^{\prime}>\min \left\{b_{s} \mid s \in g_{j}\right\}, t$ will win in the auction. Her utility will not change and is still $u_{i}^{\prime k}=v_{i}-\min \left\{b_{s} \mid s \in g_{j} \backslash\{t\}\right\}=u_{i}^{k}$ because the lowest bid of the group has not changed. But if she loses the auction her utility will be 0 .

We can see that for an elementary buyer $t$, she can not improve her utility no matter how $i$ bids, which can be indicated as: $u_{i}^{k} \geq u_{i}^{\prime k}$. As we supposed above, the elementary buyer $t$ is the $k$ th one generated from buyer $i$. For buyer $i$, her utility is

$$
u_{i}=\sum_{k=1}^{y_{i}} u_{i}^{k} \geq \sum_{k=1}^{y_{i}} u_{i}^{\prime k}=u_{i}^{\prime} .
$$


We can get the conclusion from the above analysis that bidding truthfully is the buyers' dominant strategy when participating in the auction.

Theorem 1. SHIELD satisfies incentive-compatibility.

We next show that SHIELD satisfies individual-rationality.

Lemma 2. SHIELD satisfies individual-rationality.

Proof. For an elementary buyer $t$, she can get 0 or higher utility through participating in the auction truthfully. So the utility of the buyer who is equipped with more than one radios can get 0 or higher utility too. That is to say: truthfully participating in the auction is not worse than staying outside, which can be indicated as follows:

$$
\begin{gathered}
u_{i}^{k} \geq 0\left(1 \leq k \leq y_{i}\right), \\
u_{i}=\sum_{k=1}^{y_{i}} u_{i}^{k} \geq 0 .
\end{gathered}
$$

Then the allocation mechanism satisfies individual-rationality.

Since SHIELD satisfies both incentive-compatibility and individual-rationality, we can draw the following conclusion.

Theorem 2. SHIELD is a strategy-proof channel auction mechanism.

\section{Evaluation Results}

In this section, we show the evaluation results. We compare the performance of SHIELD with that of SMALL and VERITAS.

\subsection{Metrics}

We use the following three metrics to evaluate the performance of the channel auction mechanisms.

- Utility: Utility is defined as the difference between a buyer's channel valuation and charge. As we mentioned in Sect. 3.2, a buyer may bid truthfully or untruthfully. The utility reflect the impacts of buyers' behaviors including bidding truthfully and untruthfully. We use this metric in our evaluations to verify that no buyer can increase her utility by misreporting.

- Spectrum utilization: Average number of radios allocated to each channel.

- Buyer satisfaction ratio: Buyer satisfaction ratio is the percentage of the buyers who get at least one channel in the auction. Buyer satisfaction ratio and spectrum utilization reflect the performance of a channel auction mechanism. 


\subsection{Evaluation Setup}

We now show the settings of the evaluation: we use a greedy graph coloring algorithm [37] to implement SHIELD. We assume that there are 6, 12, or 24 idle channels available and evaluate the cases in which every buyer has a single radio or 3 radios. We vary the number of buyers from 20 to 400 . The sized terrain area can be $1000 \times 1000$, $1500 \times 1500$, or $2000 \times 2000$ meters. The interference range of each node is set to 425 meters. We assume that buyers' valuation per channel are randomly distributed in $(0,1]$.

\subsection{Evaluation Results}

In our first set of evaluations, we show that, SHIELD ensures that no buyer can increase her utility by misreporting the per-channel valuation. We set the number of buyers to 200. We randomly choose a buyer to show the results of honest reporting and misreporting. Since the utilities of the buyers when bidding truthfully and untruthfully is the same in most of the cases, to illustrate clearly, we just show the cases in which these two utilities are different. The simulation is repeated more than 1000 times. Fig. 3 shows

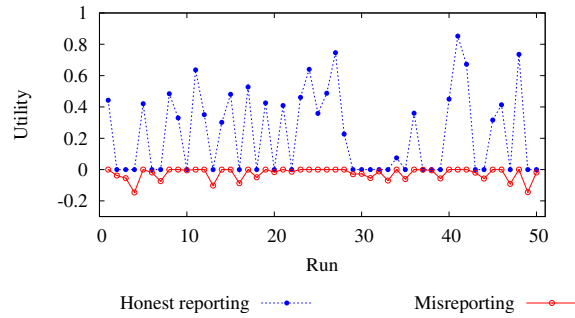

(a) Each buyer is equipped with a single radio.

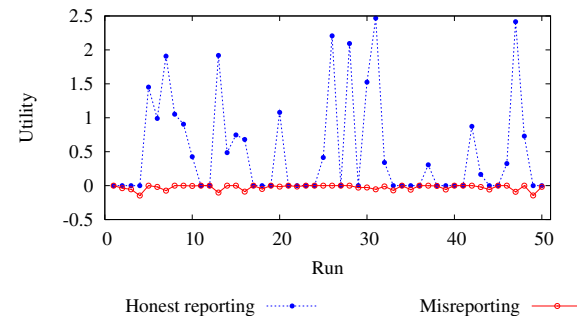

(b) Each buyer is equipped with three radios.

Fig. 3. Utilities of buyer 55 if she bids truthfully and untruthfully when each buyer is equipped with a single radio or three radios in a terrain area of $2000 \times 2000$ meters

the results of buyer 55 . Evaluation results of other nodes are similar to that of buyer 55 when bidding truthfully and untruthfully. When the two utilities are different, buyer 55 can always get a much higher utility when bidding truthfully. In Fig. 3. we can also get that when buyer 55 bids truthfully, the utility is always non-negative, while bidding untruthfully can lead to negative utility. Therefore, a buyer can not increase his utility by misreporting.

In our second set of evaluations, we compare the performance of SHIELD with SMALL and VERITAS, in terms of spectrum utilization and buyer satisfaction ratio. Our evaluation results show that SHIELD performs better than SMALL and VERITAS. When the buyers are extremely sparse, SHIELD performs a little bit worse than VERITAS. The reason for this is that SHIELD sacrifices a buyer in each winning group. As the number of buyers increases SHIELD performs better than VERITAS. SHIELD always outperforms SMALL regardless of the number of buyers and the size of terrain area.

Fig. 4 shows spectrum utilizations of SHIELD, SMALL and VERITAS under the condition that there are 6,12 , and 24 idle channels available. In this evaluation, we set 


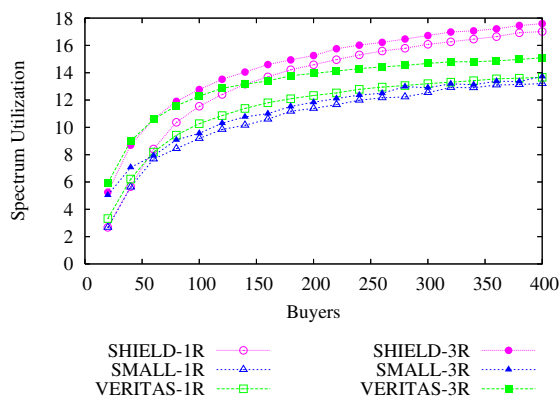

(a) There are 6 channels available.

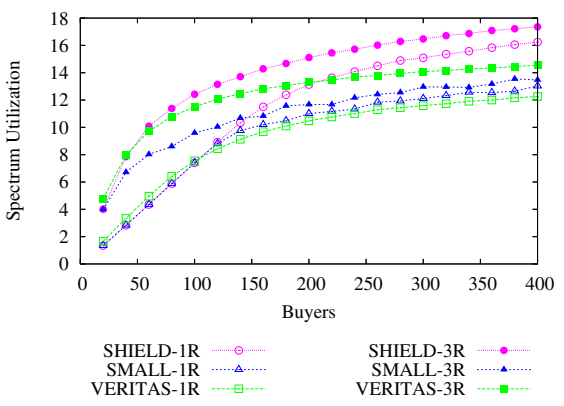

(b) There are 12 channels available.

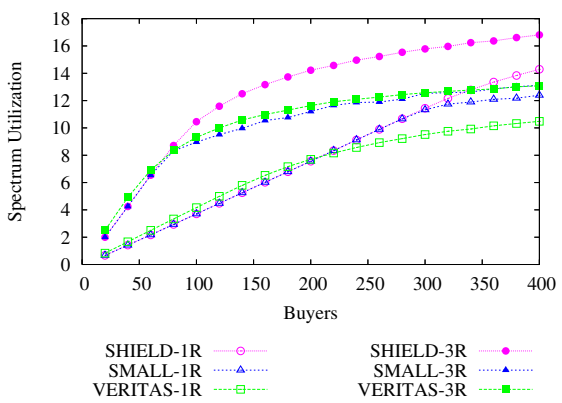

(c) There are 24 channels available.

Fig. 4. Spectrum utilizations of SHIELD, SMALL, and VERITAS when there are 6, 12, and 24 channels provided. Each buyer is equipped with one radio or three radios, in a terrain area of $2000 \times 2000$ meters.

the terrain area to $2000 \times 2000$ meters. We can see from Fig. 4 that when the number of buyers is small, SHIELD achieves a little bit lower spectrum utilization than VERITAS. This is because VERITAS does not need to sacrifice any buyer. When the number of buyers is more than a critical value(e.g. 120 in Fig. 4(a), SHIELD outperforms VERITAS. This is because non-grouping based algorithm used in VERITAS fails to fully consider the whole network topology. Fig. 4(b) and Fig. 4(c) show that, when each is equipped with three radios and the number of buyers is more than 80, SHIELD performs much better than SMALL and VERITAS. SHIELD outperforms better than SMALL in most of the cases.

Fig. 5 shows the spectrum utilizations of SHIELD, SMALL, and VERITAS in different terrain areas with the same buyer density when each buyer is equipped with a single radio or three radios. We assume there are 80,180, and 320 buyers that are randomly distributed in the terrain areas when the size of terrain area is $1000 \times 1000,1500 \times 1500$, and $2000 \times 2000$ meters, respectively. We can see from Fig. 5 that SHIELD always performs not worse than SMALL and VERITAS. Especially when the terrain area is relatively large $(1500 \times 1500$, and $2000 \times 2000$ meters $)$, SHIELD performs much better than SMALL and VERITAS.

Fig. 6 shows the buyer satisfaction ratios of SHIELD, SMALL and VERITAS. In Fig. 6(a), Fig.6(b) and Fig. 6(c), there are 6, 12, and 24 idle channels available, respectively. In each figure, we show the simulation results when each buyer requests only one radio 


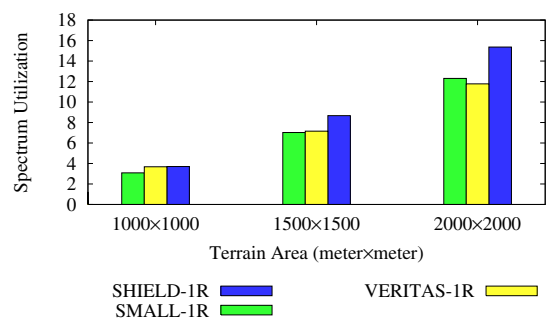

(a) Each buyer is equipped with a single radio.

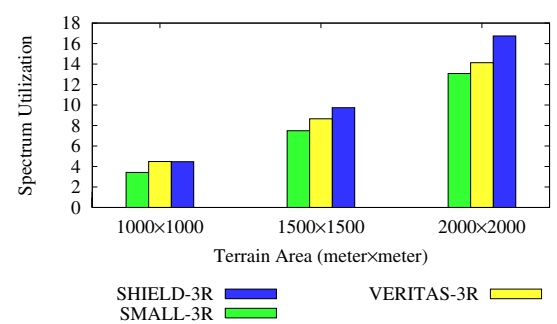

(b) Each buyer is equipped with three radios.

Fig. 5. Spectrum utilizations of SHIELD, SMALL and VERITAS for auctioning 12 channels with the same density of buyers in terrain areas with different sizes, when a buyer is equipped with one radio or three radios

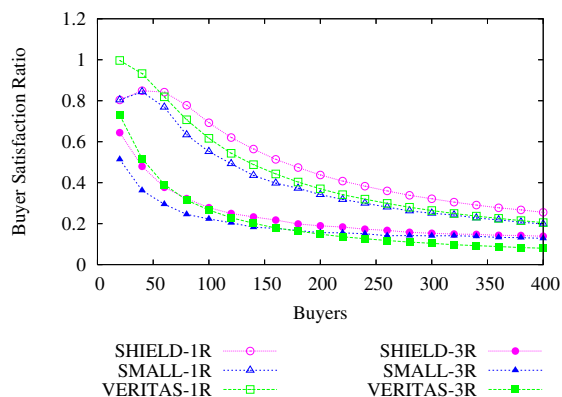

(a) There are 6 channels available.

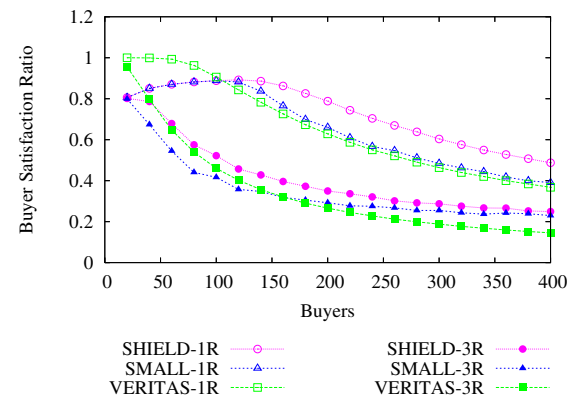

(b) There are 12 channels available.

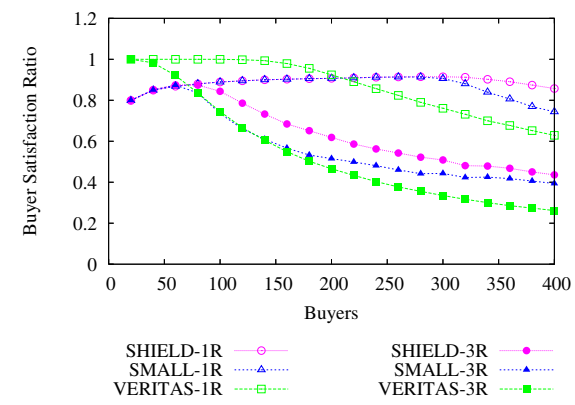

(c) There are 24 channels available.

Fig. 6. Buyer satisfaction ratios of SHIELD, SMALL and VERITAS when there are 6, 12, and 24 channels provided. Each buyer is equipped with one radio or three radios, in a terrain area of $2000 \times 2000$ meters.

or three radios in $2000 \times 2000$ meters terrain area. We can see from Fig. 6 that when the number of buyers is very small, SHIELD performs a little worse than VERITAS. The reason for this is that SHIELD sacrifices a buyer in each winning group. When the number of buyers is a little larger, SHIELD and SMALL outperforms VERITAS. When each buyer is equipped with a single radios, Fig. 6(b) and Fig. 6(c) shows that 


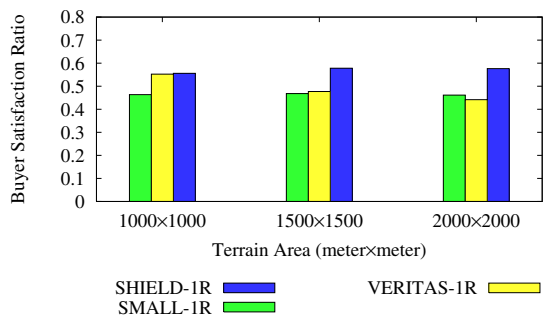

(a) A buyer is equipped with a single radio.

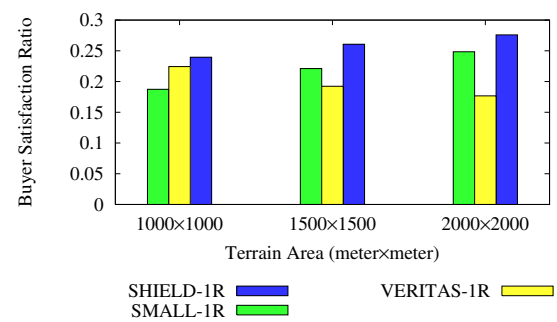

(b) A buyer is equipped with three radios.

Fig. 7. Buyer satisfaction ratios of SHIELD, SMALL and VERITAS for auctioning 12 channels with the same buyers density in terrain areas of different sizes, when a buyer is equipped with one radio or three radios

SHIELD and SMALL get nearly the same buyer satisfaction ratios when the number of buyers is less than 125 and 310, respectively. This is because SHIELD and SMALL use the same method of winner selection in one winning group. When each buyer is equipped with three radios, Fig. 6(a) and Fig. 6(b) show that SHIELD achieves higher buyer satisfaction ratios than SMALL and VERITAS in most of the cases. Fig. 6(a) and Fig. 6(b) also show that when the number of buyers is very large, SHIELD and SMALL achieves more closer buyer satisfaction ratio and they both outperforms VERITAS.

Fig. 7 shows the buyer satisfaction ratios of SHIELD, SMALL, and VERITAS for auctioning 12 channels in the same density of buyers in different terrain areas, when a buyer is equipped with one or three radios. We assume there are 80,180 , and 320 when the terrain area is $1000 \times 1000,1500 \times 1500$, and $2000 \times 2000$ meters, respectively. The buyers are randomly distributed in the terrain area. We can see from Fig. 7 that SHIELD always performs not worse than SMALL and VERITAS. Especially when the terrain area is relatively large $(1500 \times 1500$, and $2000 \times 2000$ meters $)$, SHIELD performs much better than SMALL and VERITAS.

\section{Related Works}

In this section, we review the related works on channel allocation with cooperative participants and non-cooperative participants.

\subsection{Existing Works with Cooperation Participants}

Generally, channel assignment schemes in cellular networks can be categorized as fixed channel assignment (FCA), dynamic channel assignment (DCA), and hybrid channel assignment (HCA), which is a combination of FCA and DCA. Many works have been done for wireless LANs(WLANs). For example, Mishra et al. [25] explored the use of channel hopping to improve the fairness and performance of overlapping 802.11 network deployments.

Many works about the channel allocation problems have also been done in wireless mesh networks. For instance, Kodialam and Nandagopal [20, 21] considered the problem of optimal channel assignment, scheduling and routing using a linear programming technique. Rad et al. [27] formulated joint channel allocation, interface assignment, 
and MAC problem. On the optimal problem of the network throughput, Alicherry et al. [2], Raniwala et al. [29], and Kodialam et al. [21] took the routing into account with channel allocation.

The spectrum allocation problem and improvement on this problem has been researched in many other wireless networks too. Kyasanur and Vidya [22] proposed a flow based routing and channel assignment approach for a single interface in ad-hoc networks. Vedantham et al. [32] investigated the granularity of channel assignment decisions that gives the best trade-off in terms of performance and complexity in ad-hoc networks. Ding et al. [8]studied distributed routing, relay selection, and spectrum allocation in cognitive and cooperative ad hoc networks. Authors in [19,46] study the spectrum management problem in use of cognitive radio.

\subsection{Existing Works with Non-cooperative Participants}

The related works showed in above section requests the users to cooperate with each other. While an another category of works consider the case with non-cooperative participants. Related works in this section can be divided into two sub-categories including static auction and online auction.

Static Auction. Felegyhazi et al. [13] studied Nash Equilibria in static multi-radio multi-channel allocation game. After that, Wu et al. [41] proposed a strongly dominant strategy equilibrium to improve the performance of channel allocation, and the scheme achieves the optimal system throughput in single-hop wirelwss networks. Han et al. [18] presented a distributed algorithms for simultaneous channel allocation of individual links and packet-scheduling, in Software-Defined Radio (SDR) wireless networks.

In recent years, Zhou et al. proposed TRUST [51] and VERITAS [50], which are based on spectrum auction and achieve truthfulness. TRUST is a general framework for truthful double spectrum auction, which takes both buyers and sellers into account and achieves good performance. VERITAS focuses on the buyers and the circumstances, under which buyers request multiple channels. The most closely related work is SMALL [39], which also focuses on the buyers except that it lets the seller to set reserve price to protect her interest.

$\mathrm{Xu}$ et al. [42] designed an efficient channel allocation algorithm in different cases, such that the the social efficiency can be approximately maximized. Xu et al. also designed a polynomial time approximation scheme to maximize the social efficiency. Mahmoud and Gupta [1] designed a polynomial-time truthful spectrum auction that offers a performance guarantee on the expecter revenue for Bayesian setting. Yang et al. [44] designed a truthful auction mechanism for the cooperative communication, named TASC. TASC is individually rational and budget-balanced, where wireless node can trade relay services. Wu et al. [40] studies the problem of adaptive-width channel allocation from a game-theoretic perspective and achieve higher system-wide throughput than that when system is in NE.

Online Auction. Many works have been done for online auctions. Hajiaghayi et al. [16] considered online auctions with a limited supply and presented value- and timestrategyproof mechanisms with constant efficiency- and revenue-competitiveness. Hajiaghayi et al. [17] gave a characterization for the online allocation rules that are truthfully implementable. Recently, Li [23] used a game theoretic model to increase the 
rebate incentive mechanism. Wang et al. [34] proposed TODA which is a truthful online double auction for spectrum allocation in wireless networks. Xu et al. [43] designed an efficient spectrum channel allocation and auction method for online wireless channel scheduling to decide whether to grant each user's exclusive usage and how much will be charged. Deek et al. [7] proposed a truthful online spectrum auction design called Topaz. Topaz can distribute spectrum efficiently while discouraging bidders from misreporting their bids or time report.

Game theoretic methods are also used in studying the media access problems in wireless networks and there are also other works on strategy-proofness in wireless networks. There are many examples including ad hoc networks [3, 4, 9, 30, 35, 36, 47.-49].

\section{Conclusion and Future Work}

In this section, we draw our conclusion and discuss the future work. In this paper, we have proposed SHIELD, which is a strategy-proof and highly efficient channel auction mechanism for multi-radio wireless networks. We have proven its strategy-proofness and have implemented SHIELD. Our evaluation results have shown that SHIELD can achieve higher performance compared with existing channel auction mechanisms. For future work, it will be interesting to investigate the problem of the collusion resistance in designing wireless channel auction mechanisms.

\section{References}

1. Al-Ayyoub, M., Gupta, H.: Truthful spectrum auctions with approximate revenue. In: Proceedings of 30th Annual IEEE Conference on Computer Communications (INFOCOM), Shanghai, China, pp. 2813-2821 (April 2011)

2. Alicherry, M., Bhatia, R., Li, L.: Joint channel assignment and routing for throughput optimization in multi-radio wireless mesh networks. In: Proceedings of The Eleventh International Conference on Mobile Computing and Networking (MobiCom), Cologne, Germany (September 2005)

3. Anderegg, L., Eidenbenz, S.: Ad hoc-VCG: a truthful and cost-efficient routing protocol for mobile ad hoc networks with selfish agents. In: Proceedings of the Ninth International Conference on Mobile Computing and Networking (MobiCom), San Diego, CA (September 2003)

4. Ben Salem, N., Buttyan, L., Hubaux, J.P., Jakobsson, M.: A charging and rewarding scheme for packet forwarding in multi-hop cellular networks. In: Proceedings of the Fourth ACM Symposium on Mobile Ad Hoc Networking and Computing (MobiHoc), Annapolis, MD (June 2003)

5. Cisco Visual Networking Index, Global mobile data traffic forecast update, 2010-2015, February1 (2011)

6. Clarke, E.H.: Multipart pricing of public good. Public Choice 11(1), 17-33 (1971)

7. Deek, L.B., Zhou, X., Almeroth, K.C., Zheng, H.: To preempt or not: Tackling bid and timebased cheating in online spectrum auctions. In: Proceedings of 30th Annual IEEE Conference on Computer Communications (INFOCOM), Shanghai, China, pp. 2219-2227 (April 2011)

8. Ding, L., Melodia, T., Batalama, S.N., Matyjas, J.D.: Distributed routing, relay selection, and spectrum allocation in cognitive and cooperative ad hoc networks. In: Proceedings of the Seventh Annual IEEE Communications Society Conference on Sensor, Mesh and Ad Hoc Communications and Networks SECON, Boston, Massachusetts, USA, June 21-25, pp. 484-492 (2010)

9. Eidenbenz, S., Resta, G., Santi, P.: Commit: A sender-centric truthful and energy-efficient routing protocol for ad hoc networks with selfish nodes. In: Proceedings of the 19th International Parallel and Distributed Processing Symposium (IPDPS), Denver, CO (April 2005) 
10. FCC Online Table of Frequency Allocations, November 18 (2008)

11. Federal Communications Commission (FCC), http://www. fCC.gov/

12. Federal Communications Commission Spectrum Policy Task Force, Report of the spectrum efficiency working group, November 15 (2002)

13. Félegyházi, M., Cagalj, M., Bidokhti, S.S., Hubaux, J.-P.: Non-cooperative multi-radio channel allocation in wireless networks. In: Proceedings of 26th Annual IEEE Conference on Computer Communications (INFOCOM), Anchorage, AK (May 2007)

14. Fudenberg, D., Tirole, J.: Game Theory. MIT Press (1991)

15. Groves, T.: Incentives in teams. Econometrica 41(4), 617-631 (1973)

16. Hajiaghayi, M.T., Kleinberg, R., Parkes, D.C.: Adaptive limited-supply online auctions. In: Proceedings of the ACM Symposium on Electronic Commerce (EC) (October 2004)

17. Hajiaghayi, M.T., Kleinberg, R.D., Mahdian, M.: Online auctions with reusable goods. In: Proceedings of the ACM Symposium on Electronic Commerce (EC) (October 2005)

18. Han, B., Kumar, V.S.A., Marathe, M.V., Parthasarathy, S., Srinivasan, A.: Distributed strategies for channel allocation and scheduling in software-defined radio networks. In: Proceedings of 28th Annual IEEE Conference on Computer Communications (INFOCOM), Rio de Janeiro, Brazil (April 2009)

19. Hou, Y.T., Shi, Y., Sherali, H.D.: Optimal spectrum sharing for multi-hop software defined radio networks. In: Proceedings of 26th Annual IEEE Conference on Computer Communications (INFOCOM), Anchorage, AK (May 2007)

20. Kodialam, M., Nandagopal, T.: Characterizing achievable rates in multi-hop wireless mesh networks with orthogonal channels. IEEE/ACM Transaction on Networking 13(4), 868-880 (2005)

21. Kodialam, M., Nandagopal, T.: Characterizing the capacity region in multi-radio multichannel wireless mesh networks. In: Proceedings of The Eleventh International Conference on Mobile Computing and Networking (MobiCom), Cologne, Germany (September 2005)

22. Kyasanur, P., Vaidya, N.: A routing protocol for utilizing multiple channels in multi-hop wireless networks with a single transceiver. In: Proceedings of the Second International Conference on Quality of Service in Heterogeneous Wired/Wireless Networks (QShine), Orlando, FL (August 2005)

23. Li, L.I.: Reputation, trust, and rebates: How online auction markets can improve their feedback mechanisms. Journal of Economics \& Management Strategy 19(2), 303-331 (2010)

24. Mas-Colell, A., Whinston, M.D., Green, J.R.: Microeconomic Theory. Oxford Press (1995)

25. Mishra, A., Shrivastava, V., Agrawal, D., Banerjee, S., Ganguly, S.: Distributed channel management in uncoordinated wireless environments. In: Proceedings of The Twelfth International Conference on Mobile Computing and Networking (MobiCom), Los Angeles (September 2006)

26. Osborne, M.J., Rubenstein, A.: A Course in Game Theory. MIT Press (1994)

27. Rad, A.H.M., Wong, V.W.: Joint channel allocation, interface assignment and mac design for multi-channel wireless mesh networks. In: Proceedings of 26th Annual IEEE Conference on Computer Communications (INFOCOM), Anchorage, AK, pp. 1469-1477 (May 2007)

28. Radio Administration Bureau(RAB), http://wgj .miit.gov.cn/

29. Raniwala, A., Gopalan, K., Cker Chiueh, T.: Centralized channel assignment and routing algorithms for multi-channel wireless mesh networks. ACM SIGMOBILE Mobile Computing and Communications Review (MC2R) 8(2), 50-65 (2004)

30. Srinivasan, V., Nuggehalli, P., Chiasserini, C.-F., Rao, R.: Cooperation in wireless ad hoc networks. In: Proceedings of 22nd Annual IEEE Conference on Computer Communications (INFOCOM), San Francisco, CA (April 2003)

31. Varian, H.: Economic mechanism design for computerized agents. In: USENIX Workshop on Electronic Commerce (1995)

32. Vedantham, R., Kakumanu, S., Lakshmanan, S., Sivakumar, R.: Component based channel assignment in single radio, multi-channel ad hoc networks. In: Proceedings of The Twelfth International Conference on Mobile Computing and Networking (MobiCom), Los Angeles (September 2006) 
33. Vickrey, W.: Counterspeculation, auctions and competitive sealed tenders. Journal of Finance 16(1), 8-37 (1961)

34. Wang, S., Xu, P., Xu, X., Tang, S., Li, X., Liu, X.: Toda: Truthful online double auction for spectrum allocation in wireless networks. In: Proceedings of the First IEEE International Symposium on New Frontiers in Dynamic Spectrum Access Networks, DySPAN (2010)

35. Wang, W., Eidenbez, S., Wang, Y., Li, X.-Y.: Ours-optimal unicast routing systems in noncooperative wireless networks. In: Proceedings of The Twelfth International Conference on Mobile Computing and Networking (MobiCom), Los Angeles (September 2006)

36. Wang, W., Li, X.-Y., Wang, Y.: Truthful multicast in selfish wireless networks. In: Proceedings of the Tenth International Conference on Mobile Computing and Networking (MobiCom), Philadelphia, PA (September 2004)

37. Welsh, D.J.A., Powell, M.B.: An upper bound for the chromatic number of a graph and its application to timetabling problems. The Computer Journal 10(1), 85-86 (1967)

38. West, D.B.: Introduction to Graph Theory, 2nd edn. Prentice Hall (1996)

39. Wu, F., Vaidya, N.: Small: A strategy-proof mechanism for radio spectrum allocation. In: Proceedings of 30th Annual IEEE Conference on Computer Communications (INFOCOM), Shanghai, China (April 2011)

40. Wu, F., Singh, N., Vaidya, N.H., Chen, G.: On adaptive-width channel allocation in noncooperative, multi-radio wireless networks. In: Proceedings of 30th Annual IEEE Conference on Computer Communications (INFOCOM), Shanghai, China, pp. 2804-2812 (April 2011)

41. Wu, F., Zhong, S., Qiao, C.: Globally optimal channel assignment for non-cooperative wireless networks. In: Proceedings of 27th Annual IEEE Conference on Computer Communications (INFOCOM), Phoenix, AZ (April 2008)

42. Xu, P., Li, X.-Y., Tang, S., Zhao, J.: Efficient and strategyproof spectrum allocations in multichannel wireless networks. IEEE Trans. Computers 60(4), 580-593 (2011)

43. Xu, P., Xu, X., Tang, S., Li, X.-Y.: Truthful online spectrum allocation and auction in multichannel wireless networks. In: Proceedings of 30th Annual IEEE Conference on Computer Communications (INFOCOM), Shanghai, China (April 2011)

44. Yang, D., Fang, X., Xue, G.: Truthful auction for cooperative communications. In: Proceedings of The Eleventh ACM Symposium on Mobile Ad Hoc Networking and Computing (MobiHoc). ACM, Paris (2011)

45. Yang, J.: Spatial channel characterization for cognitive radios. Master's thesis, University of California, Berkeley (2004)

46. Zheng, H., Peng, C.: Collaboration and fairness in opportunistic spectrum access. In: Proceedings of IEEE International Conference on Communications(ICC) (September 2005)

47. Zhong, S., Li, L.E., Liu, Y.G., Yang, Y.R.: On designing incentive-compatible routing and forwarding protocols in wireless ad-hoc networks-an integrated approach using game theoretical and cryptographic techniques. In: Proceedings of The Eleventh International Conference on Mobile Computing and Networking (MobiCom), Cologne, Germany (September 2005)

48. Zhong, S., Chen, J., Yang, Y.R.: Sprite, a simple, cheat-proof, credit-based system for mobile ad-hoc networks. In: Proceedings of 22nd Annual IEEE Conference on Computer Communications (INFOCOM), San Francisco, CA (April 2003)

49. Zhong, S., Wu, F.: On designing collusion-resistant routing schemes for non-cooperative wireless ad hoc networks. In: Proceedings of The Thirteenth International Conference on Mobile Computing and Networking (MobiCom), Montreal, Canada (September 2007)

50. Zhou, X., Gandhi, S., Suri, S., Zheng, H.: ebay in the sky: Strategy-proof wireless spectrum auctions. In: Proceedings of The Fourteenth International Conference on Mobile Computing and Networking (MobiCom), San Francisco, CA (September 2008)

51. Zhou, X., Zheng, H.: Trust: A general framework for truthful double spectrum auctions. In: Proceedings of 28th Annual IEEE Conference on Computer Communications (INFOCOM), Rio de Janeiro, Brazil (April 2009)

52. Zhou, X., Zheng, H.: Breaking bidder collusion in large-scale spectrum auctions. In: Proceedings of The Eleventh ACM Symposium on Mobile Ad Hoc Networking and Computing (MobiHoc), Chicago, IL (June 2010) 